\title{
Gastrostomía endoscópica percutánea en paciente con catéter de diálisis peritoneal. Reporte de caso
}

\section{Percutaneous endoscopic gastrostomy in a patient with a peritoneal dialysis catheter. Case report}

${ }^{\circ}$ Pedro Luis Imbeth Acosta ${ }^{1},{ }^{\oplus}$ Nehomar Eduardo Pájaro Galvis ${ }^{2},{ }^{\oplus}$ Gustavo Aroca Martínez ${ }^{3}$,

${ }^{\oplus}$ Donaldo Rafael Pautt Caraballo ${ }^{4}{ }^{\oplus}$ Luis Gabriel Salgado Montiel ${ }^{5},{ }^{\oplus}$ María Carolina Monterrosa Robles ${ }^{5}$

${ }^{1}$ Servicio de Gastroenterología, Clínica Blas de Lezo, Cartagena, Colombia.

${ }^{2}$ Departamento de Medicina Interna, Facultad de Ciencias de la Salud, Universidad del Sinú, Cartagena, Colombia.

${ }^{3}$ Servicio de Nefrología, Clínica de la Costa, Barranquilla, Colombia.

${ }^{4}$ Unidad de Cuidados Intensivos, Clínica Blas de Lezo, Cartagena, Colombia.

${ }^{5}$ Unidad de Cuidados Intensivos, Nuevo Hospital Bocagrande, Cartagena, Colombia.

\begin{abstract}
Resumen
La gastrostomía endoscópica percutánea (GEP), descrita por primera vez en la década de 1980, es un procedimiento universalmente utilizado para asegurar la alimentación enteral en pacientes que conservan la función del tracto digestivo, pero que por alguna condición médica o quirúrgica no pueden ingerir alimentos sólidos o líquidos. La realización de una GEP en pacientes con diálisis peritoneal (DP) sigue siendo controvertida, ya que muchos autores la consideran una contraindicación absoluta debido al alto riesgo de morbimortalidad asociada a infección intraperitoneal.

Se presenta el caso de un paciente de 70 años, con antecedente de enfermedad renal crónica en estadio terminal y portador de catéter de DP, quien requirió GEP. Se socializa la estrategia utilizada en este caso particular.

Palabras clave: enfermedad renal crónica, diálisis peritoneal, gastrostomía (DeCS).
\end{abstract}

https://doi.org/10.22265/acnef.7.2.389

\begin{abstract}
Percutaneous endoscopic gastrostomy (PEG), described in the 1980s, is a procedure universally used to ensure enteral feeding in patients who retain digestive tract function, but who cannot eat solid or liquid foods due to any medical or surgical condition. The performance of PEG in patients with peritoneal dialysis (PD) remains controversial, being considered an absolute contraindication for many authors, taking into account the high risk of morbidity and mortality associated with intraperitoneal infection. We present the case of a 70-year-old patient, with a history of chronic end-stage renal disease with a peritoneal dialysis catheter, who required PEG; and we propose a management protocol for this type of patient.
\end{abstract}

Keywords: chronic kidney disease, peritoneal dialysis, gastrostomy (MeSH).

https://doi.org/10.22265/acnef.7.2.389

\section{Introducción}

$\mathrm{L}$ a gastrostomía endoscópica percutánea (GEP) es un procedimiento que nace a raíz de la necesidad de brindar alimentación enteral de más de 4 semanas a aquellos pacientes que por disminución del estado de consciencia, condiciones neurológicas o alteración estructural y/o mecánica de la vía digestiva no pueden ingerir alimentos por vía oral. Gauderer \& Ponsky ${ }^{1}$ establecen que esta técnica fue creada por cirujanos pediátricos en 1980 debido a la necesidad de alimentar a pacientes con las alteraciones ya descritas. El procedimiento se realiza mediante una esofagogastroduodenoscopia en la que se transilumina la pared abdominal del epigastrio, a partir de lo cual se ubica sitio de punción que se infiltra con lidocaína al $2 \%$, puncionando piel hasta la cavidad gástrica y avanzando la guía, la cual se recupera con asa endoscópica; luego se procede a avanzar la sonda de gastrostomía, se verifica la posición apropiada de tope intragástrico de la misma y se verifica su permeabilidad.

Citación: Imbeth Acosta PL, Pájaro Galvis NE, Aroca Martínez G, Pautt Caraballo DR, Salgado Montiel LG, Monterrosa Robles MC. Gastrostomía endoscópica percutánea en paciente con catéter de diálisis peritoneal. Reporte de caso. Rev. Colomb. Nefrol. 2020;7(2):119-122. https://doi.org/10.22265/acnef.7.2.389 Recibido: 15.01.20; Aceptado: 30.04.20, Publicado en línea:11.08.20

Correspondencia: Nehomar Pajaro Galvis, neho94@hotmail.com 
En terapia intensiva las GEP son un procedimiento común, ya que los pacientes internados presentan una o más de las condiciones antes mencionadas y por tanto los sujetos son beneficiados de alimentación enteral temprana.

El caso reportado llama la atención porque presenta un paciente con diálisis peritoneal (DP) que se benefició de la alimentación por GEP, la cual puede llegar a ser perjudicial e incluso retrasar el reinicio de alimentación enteral en este tipo de pacientes.

\section{Presentación del caso}

Paciente masculino de 70 años de edad con antecedentes de hipertensión arterial, diabetes mellitus tipo 2 insulinorequiriente y enfermedad renal crónica estadio terminal en protocolo de DP, quien ingresó al servicio de urgencias con un cuadro clínico de aproximadamente 5 minutos de evolución consistente en deterioro del estado de conciencia secundario a crisis convulsiva tónico clónica generalizada asociada a desviación de la comisura labial hacia la izquierda, relajación de esfínteres y hemiparesia derecha. Al ingreso se realizó una tomografía de cráneo simple que mostró accidente cerebrovascular isquémico extenso en territorio de la arteria cerebral media izquierda. Debido al progresivo déficit neurológico, el sujeto requirió intubación orotraqueal.

Dado el estado del paciente, se ordenó GEP para asegurar vía de alimentación enteral y se realizó valoración en conjunto con nefrología y gastroenterología, especialidades que consideraron pertinente cambiar la modalidad de terapia de reemplazo renal, suspendiendo la DP sin retirar el catéter e implantando un catéter de hemodiálisis transitorio previo a la realización de la GEP, esto con el fin de disminuir el riesgo de infección intraabdominal.

El paciente recibió profilaxis antimicrobiana durante 24 horas con cefalosporina de primera generación y se le indicó reinicio de DP 14 días después de la realización de la GEP. En el seguimiento a los 15 días y a los 3 meses, el paciente no desarrolló complicaciones de índole infecciosa y tuvo cultivos de líquido peritoneal negativos para agentes bacterianos y fúngicos, ade- más el catéter de DP y la gastrostomía se encontraban con adecuado funcionamiento.

\section{Discusión}

La GEP es una técnica endoscópica que permite la colocación de un tubo flexible para comunicar de forma temporal o permanente la pared abdominal y la cavidad gástrica con el fin de permitir el paso de alimentos sólidos o líquidos al tubo digestivo en pacientes con imposibilidad para deglutir ${ }^{2}$. A continuación, en la Tabla 1 se resumen las indicaciones, las contraindicaciones y las complicaciones más frecuentemente asociadas a la GEP:

La GEP en pacientes con DP sigue siendo un tema controvertido debido a la alta frecuencia de complicaciones infecciosas asociadas, e inclusive por el riesgo de confundir ambos dispositivos, lo que resulta en la infusión de suplemento nutricional en la cavidad peritoneal $^{3}$. En la literatura hay poca información respecto a la implementación de GEP y DP y la mayoría de publicaciones corresponde a población pediátrica. Aun así, existen reportes de casos que muestran mayor mortalidad asociada a peritonitis en pacientes en los que se realiza GEP y poseen catéter de $\mathrm{DP}^{4-8}$. Es por esto que muchos autores clasifican la DP como una contraindicación absoluta para realizar GEP y no recomiendan su realización ${ }^{9}$.

El elevado riesgo de infección asociada a la colocación de la sonda de gastrostomía en este tipo de pacientes se debe a la comunicación inmediata de la piel con la cavidad peritoneal. Dicho riesgo disminuye con la maduración de la gastrostomía (2-4 semanas) debido a la formación de un tracto fistuloso ${ }^{2}$. En el presente caso, se describe la conducta que se tomó para este paciente, y con la cual se logró colocar la GEP de manera segura, disminuyendo el riesgo de complicaciones infecciosas.

\section{Conclusiones}

El uso de antibióticos profilácticos se justifica para la disminución de las tasas de infección periostomal. Las tasas de peritonitis fúngica y bacteriana son ma-

120 Gastrostomía endoscópica percutánea en paciente con catéter de diálisis peritoneal.

Reporte de caso 
Tabla 1. Indicaciones, contraindicaciones y complicaciones más asociadas a la gastrostomía endoscópica percutánea.

\begin{tabular}{|c|c|}
\hline Indicaciones & Contraindicaciones \\
\hline $\begin{array}{l}\text { Pacientes con enfermedades potencialmente reversibles } \\
\text { Enfermedades neurológicas (síndrome de Guillain-Barré, accidente } \\
\text { cerebrovascular, trauma craneoencefálico y anorexia nerviosa), } \\
\text { hiperémesis gravídica, quemaduras severas, politraumatismo y trauma } \\
\text { facial, trasplantes con desnutrición previa, enfermedades del esófago } \\
\text { y tumores de cabeza y cuello tratados con quimioterapia y } \\
\text { radioterapia. }\end{array}$ & $\begin{array}{l}\text { Locales } \\
\text { Patología gástrica activa, gastrectomía } \\
\text { total, obesidad extrema, laparotomía } \\
\text { previa de la línea media (dificulta } \\
\text { encontrar el sitio de punción) }\end{array}$ \\
\hline $\begin{array}{l}\text { Pacientes que tienen enfermedades irreversibles } \\
\text { Enfermedades neurológicas (esclerosis lateral amiotrófica, esclerosis } \\
\text { múltiple, demencia, enfermedad de Parkinson, enfermedad de } \\
\text { Alzheimer, accidente cerebrovascular, encefalopatía postanóxica, } \\
\text { metástasis cerebrales, tumores cerebrales, lesión cerebral - traumática } \\
\text { o quirúrgica-); poliomielitis; distrofia muscular progresiva; tumores } \\
\text { de cabeza y cuello; malformaciones faciales y neoplasias orofaríngeas, } \\
\text { del esófago y cardias; tumores de orofaringe; dermatomiositis; } \\
\text { polimiositis: amiloidosis; fibrosis quística; síndrome del intestino } \\
\text { corto; enfermedad inflamatoria intestinal, y esclerodermia. }\end{array}$ & $\begin{array}{l}\text { Contraindicaciones absolutas } \\
\text { Interposición colónica, gastrectomía } \\
\text { parcial o subtotal, ascitis masiva, } \\
\text { hipertensión portal con varices } \\
\text { gástricas, diálisis peritoneal, } \\
\text { patología gástrica activa y trastornos } \\
\text { de la coagulación. }\end{array}$ \\
\hline $\begin{array}{l}\text { Pacientes con enfermedades terminales } \\
\text { Neoplasias malignas avanzadas, sida, obstrucción intestinal por } \\
\text { carcinomatosis peritoneal, enteritis por radiación y pancreatitis aguda } \\
\text { grave. }\end{array}$ & $\begin{array}{l}\text { Complicaciones } \\
\text { Infección de la herida, fascitis } \\
\text { necrotizante, sangrado, fuga } \\
\text { periostomal, ulceración, retiro } \\
\text { involuntario de sonda de gastrostomía, } \\
\text { fuga de aliento a cavidad peritoneal, } \\
\text { neumoperitoneo, íleo, perforación } \\
\text { esofágica, fístula colocutánea, siembra } \\
\text { tumoral en sonda de gastrostomía, } \\
\text { fístula gástrica posterior a retiro de } \\
\text { sonda de gastrostomía. }\end{array}$ \\
\hline $\begin{array}{l}\text { Prevención de la desnutrición en enfermedades pediátricas. } \\
\text { Quimioterapia en enfermedad oncológica. } \\
\text { Dismotilidad orofaríngea. } \\
\text { Pacientes sometidos a radioterapia para carcinomas de cabeza y cuello }\end{array}$ & \\
\hline
\end{tabular}

Fuente: elaboración con base en Friginal-Ruiz \& Lucendo ${ }^{2}$.

yores cuanto más temprano se inicia la DP posterior a la GEP; en el presente caso no se utilizó profilaxis antifúngica y no se presentaron infecciones intraabdominales de ningún tipo. No obstante, es necesario realizar nuevos estudios con un mayor número de pacientes para evaluar adecuadamente la seguridad de esta estrategia, así como los beneficios y/o posibles efectos adversos de la profilaxis antibiótica y antifúngica.

\section{Conflicto de intereses y financiación}

Ninguno declarado por los autores.

\section{Agradecimientos}

Ninguno declarado por los autores. 


\section{Contribución de los autores}

Redaccion del caso clínico: Luis Salgado Montiel, Donaldo Pautt.

Redacción de la discusión: Gustavo Aroca, Pedro Imbett Acosta.

Algoritmo: Nehomar Pajaro Galvis, Maria Monterroza Robles.

\section{Responsabilidades éticas}

Los autores declaran que para esta investigación no se realizaron experimentos en seres humanos ni en animales.

\section{Derecho a la privacidad y consentimiento in- formado}

Los autores declaran que en este artículo no aparecen datos de pacientes.

\section{Referencias}

1. Gauderer MW, Ponsky JL, Izant RJ. Gastrostomy without laparotomy: a percutaneous edoscopic technique. J Pediatr Surg. 1980;15(6):872-5. https://dx.doi.org/10.1016/s0022-3468(80)80296-X.

2. Friginal-Ruiz AB, Lucendo AJ. Percutaneous Endoscopic Gastrostomy: A Practical Overview on Its Indications, Placement Conditions, Management, and Nursing Care. Gastroenterol Nurs. 2015;38(5):354-66. https://dx.doi.org/10.1097/SGA.0000000000000150.

3. Lew SQ, Gruia A, Hakki F. Adult Peritoneal Dialysis Patient with Tenckhoff and Percutaneous Endoscopic Gastrostomy Catheters. Perit Dial Int. 2011;31(3):360-1. https://dx.doi.org/10.3747/pdi.2010.00249.

4. Ledermann SE, Spitz L, Moloney J, Rees L, Trompeter RS. Gastrostomy feeding in infants and children on peritoneal dialysis. Pediatr Nephrol. 2002;17(4):246-50. https://dx.doi.org/10.1007/s00467-002-0846-x.

5. Ramage IJ, Harvey E, Geary DF, Hébert D, Balfe JA, Balfe JW. Complications of gastrostomy feeding in children receiving peritoneal dialysis. Pediatr Nephrol. 1999;13:249-52. https://doi.org/10.1007/s004670050603.

6. Rahim KA, Seidel K, McDonald RA. Risk factors for catheter related complications in pediatric peritoneal dialysis. Pediatr Nephrol. 2004;19(9):1021-8. https://dx.doi.org/10.1007/s00467-004-1520-2.

7. Murugasu B, Conley SB, Lemire JM, Portman RJ. Fungal peritonitis in children treated with peritoneal dialysis and gastrostomy feeding. Pediatr Nephrol. 1991;5(5):620-1. https://dx.doi.org/10.1007/BF00856656.

8. Butani L, Berg G, Makker SP. Gastrostomy tube leak in a patient receiving peritoneal dialysis. Pediatr Nephrol. 2001;16(10):787-9. https://dx.doi.org/10.1007/s004670100674.

9. Dahlan R, Biyani M, McCormick BB. High mortality following gastrostomy tube insertion in adult peritoneal dialysis patients: case report and literature review. Endoscopy. 2013;45(Suppl 2):E313-4. https://dx.doi.org/10.1055/s-0033-1344408.

122 Gastrostomía endoscópica percutánea en paciente con catéter de diálisis peritoneal.

Reporte de caso

e2500-5006 Revista Colombiana de Nefrología 